\title{
Homodyne tomography characterization and nonlocality of a dual-mode optical qubit
}

\author{
S. A. Babichev, J. Appel, A. I. Lvovsky * \\ Fachbereich Physik, Universität Konstanz, D-78457 Konstanz, Germany
}

(Dated: November 13, 2018)

\begin{abstract}
A single photon, delocalized over two optical modes, is characterized by means of quantum homodyne tomography. The reconstructed four-dimensional density matrix extends over the entire Hilbert space and thus reveals, for the first time, complete information about the dual-rail optical quantum bit as a state of the electromagnetic field. The experimental data violate the Bell inequality albeit with a loophole similar to the detection loophole in photon counting experiments.

PACS numbers: 03.65.Wj, 03.65.Ud, 03.67.Mn, 42.50.Dv
\end{abstract}

According to Feynman, single-particle interference is "a phenomenon which is impossible, absolutely impossible, to explain in any classical way, and which has it in the heart of quantum mechanics" [1]. The explanation offered by quantum mechanics is that a particle incident onto a beam splitter is not, as classically expected, randomly reflected or transmitted, but forms a delocalized coherent superposition of these two possibilities. The quantum state in the two beam splitter output modes $A$ and $B$ can be expressed as

$$
|\Psi\rangle=\tau\left|1_{A}, 0_{B}\right\rangle-\rho\left|0_{A}, 1_{B}\right\rangle,
$$

where $\tau^{2}$ and $\rho^{2}$ are, respectively, the beam splitter transmission and reflectivity, and the kets in the right-hand side are written in the particle-number basis.

In this article, we investigate the delocalized state formed by a photon. We employ homodyne tomography, a state characterization technique based on phase-sensitive measurements of the electromagnetic field quadratures. Although there is only one light particle, we find it to affect the field in both modes simultaneously, giving rise to nonclassically correlated, phase-dependent quadrature statistics. This is a direct consequence of the coherent, entangled nature of the state (11) and is remarkable because the optical phase in both the single-photon and vacuum states considered individually is completely uncertain.

We show that the delocalization of the photon in the state (11) can be formulated as a noncontextual violation of local realism, namely as a violation of Bell's inequality [2]. Nonlocality of the single photon has been discussed earlier in a number of theoretical publications, and various experiments were proposed for its demonstration 3 none of which have so far been realized. The present work achieves this goal by using a very different measurement method and by introducing an assumption which resembles the fair sampling assumption in traditional experiments on nonlocality [4]. To our knowledge, this is the first experimental verification of Bell's theorem performed in a continuous-variable setting.

Apart from its fundamental implications, our experiment finds its use in the linear optical implementation of quantum optical information processing [5], in which the state (11) plays the role of a quantum bit. To date, characterization of optical qubits has been based on studying relative photon number statistics in each mode and in their various linear superpositions as well as (in the case of multiple qubits) photon number correlations between modes. Employing this approach, White et al. have implemented tomography of entangled two-qubit systems 6]. Most recently, this technique has been extended to characterization of quantum dynamical processes [7]. Similar methods are used in celebrated quantum cryptography and quantum teleportation protocols.

A major drawback of the photon counting approach to quantum state characterization is the a priori assumption that the modes involved are either in one of the states $\left|1_{A}, 0_{B}\right\rangle,\left|0_{A}, 1_{B}\right\rangle$ or in their linear combination. As a result, being characterized are not the quantum states of the carrier modes, but their projections onto a subspace spanned by these basis vectors. It is neglected that in actual experimental situations, most of the time the state of the modes does not belong to this subspace but is the double vacuum $\left|0_{A}, 0_{B}\right\rangle$. These overwhelming events, as well as all other events not falling into the qubit frame, were simply eliminated from the analysis.

The homodyne tomography method employed in this paper is free from this disadvantage as it permits complete characterization of a quantum optical ensemble. So far it has been employed to study single- [8] and multimode [9] squeezed states and, more recently, the singlephoton and related states [10]. Here we apply this technique to the delocalized photon state (11) and evaluate its density matrix and its Wigner function. A detailed theoretical analysis of different aspects of an experiment such as ours was made by Jacobs and Knight 11] as well as Grice and Walmsley 12].

In our experiment, the initial single-photon state was prepared by means of a conditional measurement on a biphoton produced via parametric down-conversion [13]. We used frequency-doubled 2-ps pulses from a modelocked Ti:Sapphire laser running at $\lambda=790 \mathrm{~nm}$ which underwent down-conversion in a BBO crystal, in a type-I frequency-degenerate configuration [10].

Field quadratures of the delocalized photon ensemble were measured by means of two homodyne detec- 
tors (associated with fictitious observers Alice and Bob) placed into each beam splitter output channel (Fig. 1(a)). The local oscillator fields were provided by the master Ti:Sapphire laser and contained several million photons per pulse. Their optical mode had to be matched to those of the state under investigation [14]. A specially designed balanced detector employed two Hamamatsu S3883 photodiodes of a 94-\% quantum efficiency whose photocurrents were directly subtracted [15]. The amplification circuit provided suppression of the electronic noise in a large frequency range from $\mathrm{DC}$ to $2-3 \mathrm{MHz}$, which enabled time-resolved quadrature measurements for each pulse. The time-integrated homodyne detector output directly corresponded to a quadrature value, with a scaling reference obtained from the vacuum state shot noise.

With every incoming photon, both detectors made a measurement of the field quadrature $X_{A}$ and $X_{B}$ with the local oscillators' phases set to $\theta_{A}$ and $\theta_{B}$, respectively. The quadrature statistics collected at various phases were used to reconstruct the density matrix of the generated ensemble.

Generally, homodyne tomography reconstruction of a two-mode state requires fine control of both local oscillator phases involved. However, the particular state studied in the present experiment was generated by splitting a single photon which has no optical phase. The sum of Alice's and Bob's phases $\theta_{A}+\theta_{B}$ is therefore meaningless and does not affect the homodyne statistics. Therefore, we let this phase vary randomly and controlled only the relative phase $\delta \theta=\theta_{A}-\theta_{B}$ of the two modes. During an experimental run, we varied this parameter slowly over a $2 \pi$ range, and acquired a few hundred thousand pairs $\left(X_{A}, X_{B}\right)$ from the homodyne detectors. We have executed two data acquisition runs using two different beam splitters with transmissions $\tau^{2}$ equal to 0.5 and 0.08 .

Fig. 1(c,d) shows histograms of the dual-mode quadrature measurements. The two-dimensional distribution $\operatorname{pr}_{\delta \theta}\left(X_{A}, X_{B}\right)$ indicates the probability of detecting a particular pair $\left(X_{A}, X_{B}\right)$ of quadratures at a given local oscillator phase setting. These densities are the marginal distributions of the four-dimensional Wigner function of the two-mode ensemble being measured. They can be used for its direct reconstruction via the inverse Radon transformation.

In this work, however, he have used a more precise maximum-likelihood reconstruction technique 16, 17]. We applied the iterative algorithm described in [18] to the two-mode density matrix of the reconstructed ensemble in the Fock representation:

$$
\hat{\rho}=\sum_{k, l, m, n=0}^{\infty} \rho_{k l m n}\left|k_{A}, l_{B}\right\rangle\left\langle m_{A}, n_{B}\right|,
$$

with the photon number restricted to five per mode. The positive operator-valued measure (POVM) operator, used to describe the homodyne detector, was modified
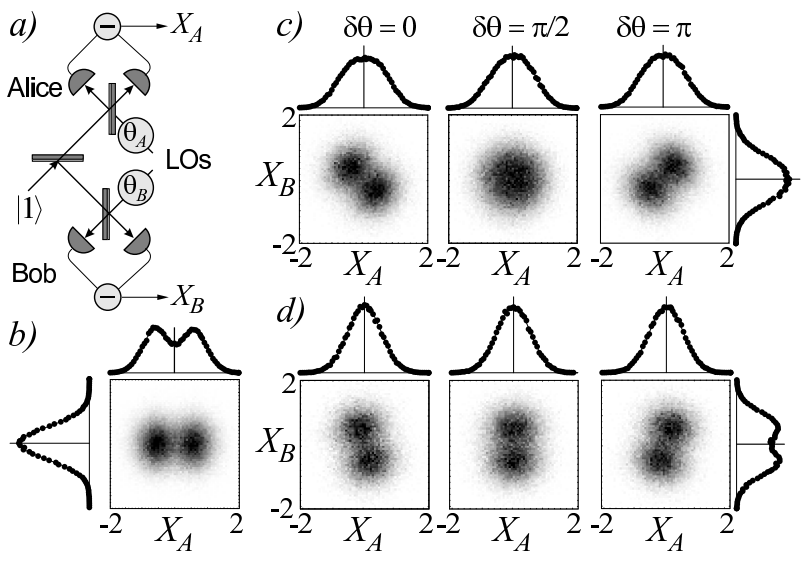

FIG. 1: (a) Scheme of the experimental setup. LOs: local oscillators. (b-d) Histograms of the experimental quadrature statistics $\operatorname{pr}_{\delta \theta}\left(X_{A}, X_{B}\right)$ for the "zero reflectivity" (b), symmetric (c), and highly reflective $(92 \%, \mathrm{~d})$ beam splitters. Phase-dependent quadrature correlations are a consequence of the entangled nature of the state $|\Psi\rangle$. Also shown are individual histograms of the data measured by Alice and Bob, which are phase-independent.

with respect to that of an ideal homodyne detector in two ways. First, we corrected for the non-perfect detection efficiency associated with linear optical losses and nonunity quantum efficiency of the homodyne detector photodiodes. These losses amounted to $1-\eta_{\text {det }}=0.14$ and are described mathematically in the form of a Bernoulli transformation affecting every photon entering the detector 17, 18, 19].

Secondly, due to a random variation of the phase $\theta_{A}+\theta_{B}$, all the POVM elements, for which $m+n \neq k+l$, vanish, and so do the respective elements of the reconstructed density matrix - akin to the off-diagonal terms of any phase-randomized single-mode ensemble.

The reconstructed density matrix (Fig. 2) features a strong contribution of the double-vacuum term $|0,0\rangle\langle 0,0|$. This is a consequence of imperfect preparation of the initial single photon: instead of the state $|1\rangle$, a statistical mixture $\hat{\rho}_{|1\rangle}=\eta|1\rangle\langle 1|+(1-\eta)| 0\rangle\langle 0|$ is available at the beam splitter input [10, 14]. The vacuum fraction is directly transferred to the dual-mode ensemble: $\hat{\rho}=\eta|\Psi\rangle\langle\Psi|+(1-\eta)| 0,0\rangle\langle 0,0|$. The reconstructed ensemble is in excellent agreement with the above equation and corresponds to the preparation efficiency $\eta=0.64$.

Some insight into the quadrature dynamics of the nonlocal single-photon state can be gained by analyzing its Wigner function (WF). Theoretically, the transformation of a quantum ensemble by a beam splitter can be expressed as a rotation of its WF in the four-dimensional phase space [19]:

$$
\begin{aligned}
& W\left(X_{A}, P_{A}, X_{B}, P_{B}\right)=W_{i n}\left(\tau X_{A}+\rho X_{B},\right. \\
& \left.\tau P_{A}+\rho P_{B}, \tau X_{B}-\rho X_{A}, \tau P_{B}-\rho P_{A}\right) .
\end{aligned}
$$




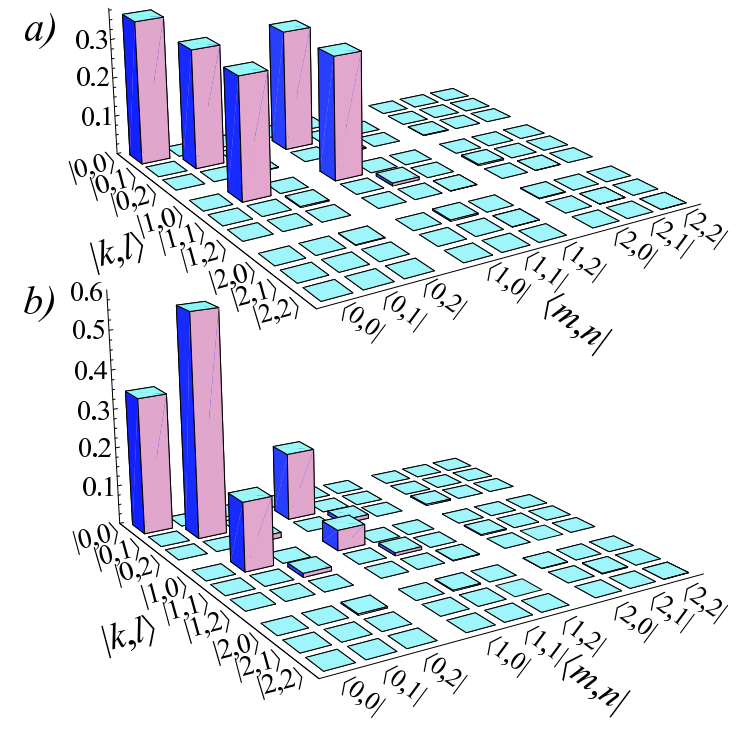

FIG. 2: Density matrix (absolute values) of the measured ensemble for the symmetric (a) and highly reflective (b) beam splitters in the photon number representation (2).

In our experiment, the input WF is an uncorrelated product of the Wigner functions of the single-photon and vacuum states in the beam splitter input modes:

$$
W_{\text {in }}\left(X_{1}, P_{1}, X_{2}, P_{2}\right)=W_{|1\rangle}\left(X_{1}, P_{1}\right) \times W_{|0\rangle}\left(X_{2}, P_{2}\right) .
$$

A rotation in the phase space entangles the input mode, generating the ensemble $|\Psi\rangle$. The effect of this rotation can be seen in Fig. 3 which shows the cross-sections of the four-dimensional WF of the reconstructed ensemble through two different planes. As expected for the single photon, the WF is negative in the phase space origin, albeit the negativity is reduced due to a non-perfect preparation efficiency.

A similar argument can be used to understand the shape of the measured quadrature probability densities. Consider an experiment in which the beam splitter has zero reflectivity, so that Alice measures the field quadratures of the single-photon state, and Bob, at the same time, those of the vacuum state. The joint quadrature statistics are, naturally, uncorrelated [Fig. 1(b)]. However, if the two modes are subjected to a beam splitter transformation prior to their measurement, their field amplitudes will mix, the quadrature histogram will rotate by an angle $\tan ^{-1}(\tau / \rho)$ and become correlated [Fig. 1(c,d)]:

$$
\begin{aligned}
& X_{A}=\tau X_{1}+\rho X_{2} \\
& X_{B}=-\rho X_{1}+\tau X_{2} .
\end{aligned}
$$

The above relation is valid only if Alice and Bob measure the same quadrature of the incoming fields, i.e. for $\delta \theta=0$ or $\delta \theta=\pi$. On the other hand, the setting
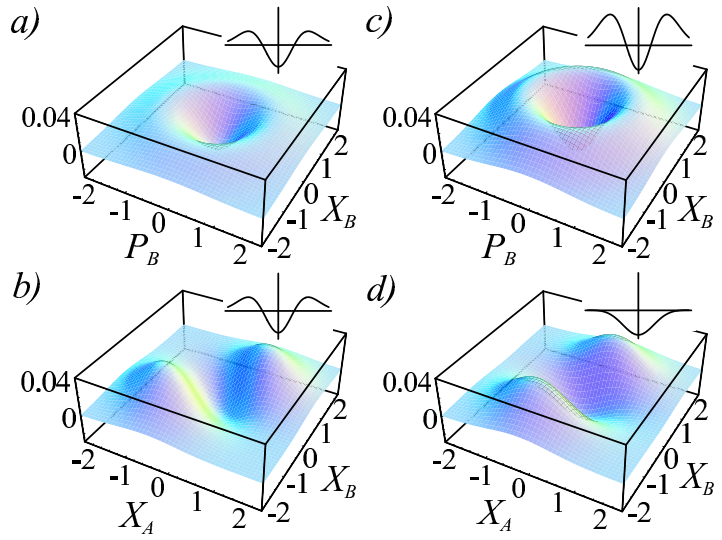

FIG. 3: Cross-sections of the two-mode Wigner function $W\left(X_{A}, X_{B}, P_{A}, P_{B}\right)$ of the reconstructed ensemble for the symmetric (left column) and highly reflective (right column) beam splitters. $(\mathrm{a}, \mathrm{b})$ : cutting plane $\left(X_{A}=P_{A}=0\right)$; the WF is axially symmetric. (c, d): cross-sections through the plane $\left(P_{A}=P_{B}=0\right)$ possesses mirror symmetry with symmetry planes oriented at an angle $\tan ^{-1}(\rho / \tau)$ with respect to the coordinate axes. Insets: cross-sections through $X_{B}=0$.

$\delta \theta=\pi / 2$ corresponds to a measurement of orthogonal quadratures, e.g. $X$ by Alice and $P$ by Bob. It can be interpreted as a simultaneous, unprecise measurement of the canonically conjugated position and momentum quadratures of the input single-photon state with an eight-port homodyne detector 19, 20]. Furthermore, the distribution $\operatorname{pr}_{\pi / 2}\left(X_{A}, X_{B}\right)$ acquired with a symmetric beam splitter [Fig. 1(c), middle] is equal to the $Q$ function of this state [21].

As discussed above, these phase-dependent features of the quadrature statistics are incompatible with a naive picture according to which the photon is randomly localized in one of the beam splitter output channels. The nonlocal character of our measurements can be formalized as a violation of the Bell inequality [2]. In order to apply the Bell theorem to the continuous field quadratures measured in this experiment, the latter had to be converted to a dichotomic format. We proceeded as follows. Suppose the output of each homodyne detector is processed by a discriminator producing the value $S=1$ if the observed quadrature exceeds a certain threshold $T, S=-1$ if it is below $-T$, and generating no output otherwise. Restricting to the events in which both discriminators have generated a value, we studied correlations $E_{A B}=<S_{A} S_{B}>$ between the values acquired by Alice and Bob.

The correlation $E_{A B}$ as a function of $\theta_{A}-\theta_{B}$ exhibits quantum interference [Fig. 4] which is similar to that observed in Bell's Gedankenexperiment for the spin correlation as a function of the angle between the axes of the Stern-Gerlach apparata. Increasing the threshold $T$ leads to a higher visibility of the interference pattern. For 

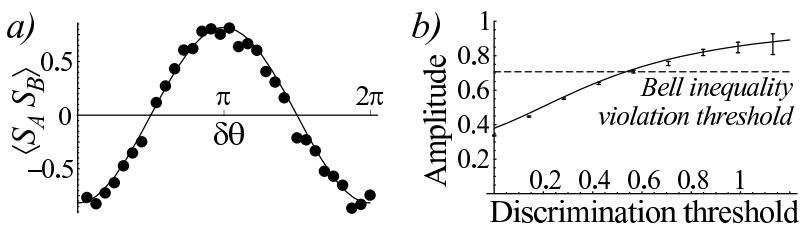

FIG. 4: Bell inequality violation by a threshold-selected subset of the experimental data [symmetric beam splitter]. (a) Interference pattern for the threshold of $T=0.85$ shows an amplitude of $0.818 \pm 0.018$; (b) theoretical and experimental dependence of the amplitude on the threshold value. A higher threshold increases the amplitude, but also reduces the number of selected data.

$T>0.54$, the amplitude exceeds $1 / \sqrt{2}$ and the Bell inequality is violated. Interpreting our data in this manner, we observed violations by up to six standard deviations.

One might object that such an interpretation involves rejection of some of the the acquired data. This objection is valid; however, similar selection is also a part of the traditional way of verifying Bell's theorem by studying the counting statistics of a polarization-entangled photon pair [4]. Indeed, every single-photon counter has a discriminator as a part of its electronic circuit, and the signals not exceeding the discrimination threshold are disregarded. Viewed outside of a context of any particular physical theory, both tests possess an equal degree of validity: they both involve a fair sampling assumption and thus suffer from the detection loophole 22].

Is our entire data set noncontextually nonlocal? Although a number of schemes of reformulating Bell's theorem for continuous variables 23. have been proposed, we have not yet found one that could be applied to our original data. On the other hand, we could not come up with a local hidden variable model that would replicate the quadrature statistics observed in this experiment 24].

In summary, we have characterized a two-mode optical qubit using homodyne tomography, reconstructing its density matrix and the Wigner function. For the first time, complete information about this quantum ensemble is revealed, including those terms that do not belong to the qubit subspace of the Hilbert space. The experimental data demonstrate a nonlocal character of the delocalized single photon but the question of noncontextual nature of this nonlocality remains open.

We are grateful to A. M. Steinberg, who asked about a possibility of selecting a data subset that violates Bell's inequality, and to J. Ries and B. Brezger for helpful discussions. We are supported by the Deutsche Forschungsgemeinschaft and the Optik-Zentrum Konstanz.

\footnotetext{
[*] Email: Alex.Lvovsky@uni-konstanz.de
}

[1] R. Feynman, R. Leighton, and M. Sands, The Feynman Lectures on Physics (Addison-Wesley, Reading, Mass., 1963, Vol. 1, p. 37

[2] J. S. Bell, Physics 1, 195 (1964).

[3] S. M. Tan, D. F. Walls, and M. J. Collett, Phys. Rev. Lett. 66, 252 (1991); K. Banaszek and K. Wodkiewicz, ibid. 82, 2009 (1999); K. Jacobs and P. L. Knight, Phys. Rev. A 54, 3738 (1996).

[4] S. J. Freedman and J. F. Clauser, Phys. Rev. Lett. 28, 938 (1972); A. Aspect, J. Dalibard, and G. Roger, Phys. Rev. Lett. 49, 1804 (1982).

[5] E. Knill, R. Laflamme, G. J. Milburn, Nature 409, 46 (2001); J. L. O'Brien et al., Nature 426, 264 (2003).

[6] A. G. White, D. F. V. James, P. H. Eberhard, and P. G. Kwiat, Phys. Rev. Lett. 83, 3103 (1999).

[7] M.W. Mitchell, C.W. Ellenor, S. Schneider, and A.M. Steinberg, Phys. Rev. Lett. 91, 120402 (2003); J. B. Altepeter et al., Phys. Rev. Lett. 90, 193601 (2003).

[8] D. T. Smithey et al., Phys. Rev. Lett. 70, 1244 (1993); G. Breitenbach, S. Schiller, and J. Mlynek, Nature 387, 471 (1997).

[9] M. Vasilyev et al., Phys. Rev. Lett. 84, 2354 (2000).

[10] A. I. Lvovsky et al., Phys. Rev. Lett. 87, 050402 (2001); A. I. Lvovsky , J. Mlynek, Phys. Rev. Lett. 88, 250401 (2002); A. I. Lvovsky, S. A. Babichev, Phys. Rev. A 66, 011801 (2002).

[11] K. Jacobs and P. L. Knight, Phys. Rev. A 54, 3738 (1996).

[12] W. Grice and I. A. Walmsley, J. Mod. Opt. 43, 795 (1996).

[13] C. K. Hong and L. Mandel, Phys. Rev. Lett. 56, 58 (1986); P. Grangier, G. Roger, and A. Aspect, Europhys. Lett. 1, 173 (1986).

[14] T. Aichele, A. I. Lvovsky, and S. Schiller, Eur. Phys. J. D 18, 237 (2002);

[15] H. Hansen et al., Opt. Lett. 26, 1714 (2001).

[16] J. Řeháček, Z. Hradil, and M. Ježek, Phys. Rev. A 63, 040303 (2001).

[17] K. Banaszek, G. M. D'Ariano, M. G. A. Paris, and M. F. Sacchi, Phys. Rev. A 61, 010304 (1999).

[18] A. I. Lvovsky, quant-ph/0311097

[19] U. Leonhardt, Measuring the Quantum State of Light, Cambridge University Press, 1997.

[20] N. G. Walker and J. E. Caroll, Electronics Letters 20, 981 (1984).

[21] U. Leonhardt and H. Paul, Phys. Rev. A 47, 2460 (1993).

[22] P. Pearle, Phys Rev. D 2, 1418 (1970).

[23] J. W. Wu, Phys. Rev. A 61, 022111 (2000); Z.-B. Chen, J.-W. Pan, G. Hou, and Y.-D. Zhang, Phys. Rev. Lett. 88, 040406 (2002); S. Mancini, V. I. Manko, E. V. Shchukin, and P. Tombesi, J. Opt. B 5, S333 (2003); J. Wenger et al., Phys. Rev. A 67, 012105 (2003).

[24] As argued by J. S. Bell in Speakable and unspeakable in quantum mechanics (Cambridge University Press, Cambridge, 1987), if the Wigner function of a two-mode ensemble is positive definite, it can automatically serve as a local hidden variable model for a homodyne measurement. In our experiment, the cumulative quantum efficiency is $\eta \eta_{\text {det }}=0.55>1 / 2$, so the WF is negative in the phase space origin, even if no correction for the detection inefficiency is made when reconstructing the state. 